\title{
Categorical interoception and the role of threat
}

Nadia Zacharioudakis ${ }^{1}$, Elke Vlemincx ${ }^{1,2}$, Omer Van den Bergh ${ }^{1}$

${ }^{1}$ Research Group Health Psychology, University of Leuven, Tiensestraat 102, 3000

Leuven, Belgium

${ }^{2}$ Department of Biological and Experimental Psychology, Queen Mary University of London, Mile End Road, London, E1 4NS, United Kingdom

Corresponding author: Omer Van den Bergh, Health Psychology, Faculty of Psychology and Educational Sciences, University of Leuven, Tiensestraat 102, Box 3726, 3000 Leuven, Belgium. E-mail: omer.vandenbergh@ kuleuven.be, T: +32 (0)16 326058.

Author e-mail addresses:

Nadia Zacharioudakis: nadia.zacharioudakis@kuleuven.be

Elke Vlemincx: e.vlemincx@qmul.ac.uk

Omer Van den Bergh: omer.vandenbergh@ kuleuven.be

Field Code Changed 


\section{Highlights}

- Categorization of interoceptive (respiratory) sensations changes perception

- Making categories threat-relevant decreased within-category discrimination, especially within a high-intensity category

- These findings suggest that interoceptive fears impact perception of interoceptive sensations 


\section{Abstract}

Interoceptive fears and biased interoception are important characteristics of somatic symptom disorders. Categorization of interoceptive sensations impacts perception of their intensity and unpleasantness. In this study we investigated whether making interoceptive categories threat-relevant further biases interoception of individual sensations compared to safe categories. Either a category containing low- or high-intensity stimuli was made threat-relevant by instructing (and occasionally experiencing) that interoceptive sensations could be followed by an unpredictable electrocutaneous stimulus. We replicated that categorization had a profound impact on perceived interoceptive sensations, with stimuli within categories being perceived as more similar than equidistant stimuli at the category border. We found some evidence for the impact of threat on perceived characteristics of stimuli (with the direction of these effects depending on whether interoceptive stimuli of low or high intensity were threat-relevant), but not for altered categorical choice behaviour. These results imply that the perception of respiratory stimuli is influenced strongly by top-down processes such as categorization, and suggest that interoceptive processing may flexibly adapt to contextual factors such as threat in healthy individuals. However, inflexible responding to repeated and/or severe threat to the internal body may compromise accurate interoception and may result in interoceptive illusions contributing to medically unexplained symptoms and syndromes.

Keywords: interoception; categorization; threat; anxiety 
1. Introduction

Medically unexplained symptoms (i.e., symptoms in the absence of any physiological dysfunction) and somatic symptom disorders are highly prevalent both in primary (see Haller et al., 2015 for a review) as well as secondary health care settings (Nimnuan et al., 2001; Reid et al., 2001), and put a high economic burden on society (Barsky et al., 2005; Bermingham et al., 2010; Hiller et al., 2003). Interoceptive sensations inform us about the state of our physiological body and motivate behaviour (e.g., eating behaviour, care seeking). However, most individuals are not good in perceiving internal stimulation (Zamariola et al., 2018), and persons with persistent medically unexplained symptoms tend to be interoceptively less accurate (Van den Bergh et al., 2017). Even though this research suggests that the correspondence between peripheral physiology and reported sensations is on average rather low, the idea that symptoms have a physiological cause is deeply rooted in our health care system. Moreover, some individuals develop clusters of multiple, persistent, and debilitating unexplained symptoms together with excessive thoughts, feelings, or behaviours related to these symptoms (i.e., somatic symptom disorder), leading to considerable psychological burden due to stigma, lack of a clear treatment, and poor prognosis (Nettleton, 2006). Even though these patients benefit from non-pharmacological treatments (such as CBT) (Clarke, 2016; Smith et al., 2015), it is unclear which aspects of existing treatments work (Henningsen et al., 2018). In order to optimize diagnostic and treatment procedures, as well as improve patients' well-being, it is thus of great importance to gain insight in the perceptual mechanisms underlying the experience of interoceptive sensations, as well as in the circumstances under which they relate or dissociate from peripheral physiological activity.

In accordance with recent theorizing about interoception and symptom perception within a predictive processing perspective, we assume that the brain makes sense of the 
constant stream of interoceptive stimulation by implicitly making categorical predictions about the sources of the stimulation (Petersen et al., 2015b; Rigoli et al., 2017; Van den Bergh et al., 2017). By grouping patterns of interoceptive stimulation into categories (e.g., cardiac vs. gastrointestinal sensations, benign sensations vs. symptoms), likely causes of the stimulation can be inferred and, if necessary, coping behaviour can be instigated. Categorization reflects an (implicit) decision process based on the likelihood of certain categorical prior beliefs, given an individual's learning history and the current context (Lynn and Barrett, 2014; Rigoli et al., 2017). For example, patterns of respiratory stimulation during exercise may be categorized as benign sensations in a healthy individual with relative certainty, but as symptoms of a disease in a person with exerciseinduced asthma (possibly inducing coping behaviours). In both persons, however, the same stimulation will likely be categorized differently when experienced in another context (e.g., in rest). Obviously, also the type and pattern of the actual somatic input plays a role: stimulation associated with suffocation (e.g., bronchoconstriction, which is typically seen in asthma) is a more reliable source of evidence to suggest a specific category than is the vague and widespread stimulation elicited by exercise (e.g., increased heart rate, blood pressure, breathing rate and depth, body temperature, ...). The relative reliability of categorical prior beliefs versus that of actual physiological input determines their relative contribution to the perception of sensations (Henningsen et al., 2018; Van den Bergh et al., 2017). If highly precise categorical prior beliefs determine interoception without being sensitive to actual somatic input, perceived sensations may be an inaccurate representation of the state of the body, which in extreme cases may take the form of interoceptive illusions (such as medically unexplained symptoms).

Importantly, anxiogenic traits and anxiety disorders have been linked to a number of cognitive biases which, we believe, are expressions of strong prior beliefs dominating 
information processing (Barrett, 2017). Anxiety is related to a tendency to expect negative and threatening events (for a review, see Bar-Haim et al., 2007) and biased fear learning, that is, difficulties in discriminating between threat and safety signals (Lissek et al., 2009) and the spread of fear from threatening to similar, but safe signals (for a review, see Dymond et al., 2015). In other words, perception in anxious individuals seems to be strongly impacted by predictions of threat resulting in lower differentiation between threat and safety signals (Paulus and Stein, 2010). Furthermore, anxiety has been related to a reduced ability to learn that signals predict safety (Lissek et al., 2009, 2005), reflecting difficulties in falsifying threat-related predictions. Since dispositional anxiety and specific interoceptive fears are higher in patients reporting medically unexplained symptoms and in patients with somatic symptom disorder (Geisser et al., 2003; Henningsen et al., 2003; Jerndal et al., 2010; Kroenke, 2003; Nijs et al., 2013; Peters et al., 2000), we assume that anxiety increases the likelihood of processing interoceptive stimulation in the light of general threat-related prior beliefs, such as bodily symptoms. When aggravated by difficulties to falsify such inaccurate predictions, a vicious circle of inaccurate interoception and possibly even interoceptive illusions may ensue.

The impact of categories on interoception was indeed found in earlier interoceptive categorization studies in which participants memorized category membership of different interoceptive stimuli (inspiratory resistances requiring more or less inspiratory effort). Allocating a set of stimuli to one category resulted in more perceived similarity and worse discrimination between stimuli belonging to that category, compared to between (equidistant) stimuli belonging to different categories (Petersen et al., 2015a, 2014), and these effects were more pronounced with higher levels of symptom reporting. The latter factor was also associated with a tendency to classify ambiguous low-intensity sensations into a high-intensity category (Petersen et al., 2015a). 
Furthermore, a number of studies using oesophageal and proprioceptive stimulation found that fear associated with low-intensity, non-painful stimuli increased the likelihood of these stimuli to be classified as 'high-intensity' or 'painful' (Zaman et al., 2018, 2017) and was related to reduced discrimination between stimuli of different intensities (compared to when fear-inducing stimulus was never paired with oesophageal stimulation) (Zaman et al., 2016). These findings suggest that individual differences in symptom reporting levels and interoceptive fear learning are related to differences in categorization of physiological stimulation, as well as the detail with which interoceptive stimulation is perceived, and may thus be important factors in the gradual uncoupling of body physiology and subjective reports thereof. We do not know, however, whether threat-related prior beliefs are weighted more in interoception, thereby being a possible mechanism relating individual differences and fear learning to biased interoception (and possibly symptom overperception). The aim of this study was to replicate the above interoceptive categorization effects and to examine whether associating threat to a specific categorical prior belief modified these effects (i.e., changed their weight in the perceptual process). Participants categorized interoceptive stimuli into a low- or highintensity category, one of which was threat-relevant in one part of the task. We expected that categorization would lead to within-category loss of perceptual detail (i.e., reduced perceived differences within, compared to between categories), and that this effect was stronger within the threat-relevant category. Furthermore, we expected that threatrelevance would result in higher importance of, and thus better, discrimination between threat and safety signals. We also expected that threat-relevance would lead to changes in the subjective border between categories. Finally, we explored effects of individual differences in anxiogenic traits. 
2. Materials and methods

2.1 Participants

Eighty-six female students $\left(M_{\text {age }}=21.06, S D_{\text {age }}=4.09\right.$, range 17-46) participated in return for course credits or monetary compensation $(€ 12)$. Participants were recruited via advertisements at the Faculty of Psychology and Educational Sciences. Since sex differences in interoceptive abilities (Grabauskaite et al., 2017; Miller and Davenport, 2015) and anxiogenic traits and fear learning are often reported (McLean and Anderson, 2009; Merz et al., 2018), and the majority of students at the faculty are female, we only included female participants. Participants were randomly allocated to a between-subjects condition (low- vs. high-intensity stimuli are associated with threat). Within these conditions, the order in which task phases were presented (first threat vs. safe phase) was also randomised. Both operations were based on a randomisation list. Data of six participants were excluded before analyses due to technical difficulties $(\mathrm{N}=4)$ or failure to adhere to task instructions $(\mathrm{N}=2)$. Exclusion criteria were a history of or current cardiovascular, respiratory, or neurological condition, medical conditions that affect respiratory capacity, acute illness, past or current psychiatric diagnosis, regular medication use (except contraceptives), having an electronic implant, pain or problem located at the wrist, request from general practitioner to avoid stressful situations, pregnancy, or uncorrected eyesight problems. All participants signed informed consent. The study was approved by the Social and Societal Ethics Committee of the University of Leuven.

\subsection{Apparatus}

The Powerbreathe K5 device (POWERbreathe International Ltd., Southam, UK) was used to present eight inspiratory resistances $\left(7,9,12,16,21,28,37\right.$, and $\left.49 \mathrm{cmH}_{2} \mathrm{O}\right)$ 
increasing in intensity with a constant factor of $32 \%$, each level requiring more effortful inhalation. Resistances were presented using Breathelink software. Inspiratory flow was registered with a pneumotachograph (Hans Rudolph Inc., Shawnee, USA) connected to the breathing device, in order to present electrocutaneous stimuli directly at the start of inhalation and to calculate categorization response times relative to the start of inhalation. Inspiratory flow was sampled and stored at $1000 \mathrm{~Hz}$.

Electrocutaneous stimuli $(2 \mathrm{~ms})$ were delivered at the left wrist via a reusable bar electrode (8 $\mathrm{mm}$ diameter, $30 \mathrm{~mm}$ spacing) filled with K-Y jelly and a Digitimer DS7A constant current stimulator (Digitimer Limited, Hertfordshire, UK).

\subsection{Self-report measures}

\subsubsection{Trait measures}

The Dutch version of the Positive and Negative Affect Schedule (Watson et al., 1988) was used to measure negative affectivity. Participants indicated how often they experience 10 negative affective states in general on a scale from 1 (seldom) to 5 (very often). The questionnaire has excellent construct validity, good internal consistency and acceptable test-retest reliability (Engelen et al., 2006; Watson et al., 1988). Participants rated anxiety in 14 suffocation-related situations on the Dutch version of the Fear of Suffocation Scale (Van Diest et al., 2010) on a scale from 0 (not anxious at all) to 4 (extremely anxious). The subscale has good construct validity, internal consistency and test-retest reliability (Radomsky et al., 2001; Van Diest et al., 2010). The Dutch version of the Intolerance of Uncertainty Scale (de Bruin et al., 2006) was used to measure participants' cognitive, emotional, and behavioural reactions to (perceived) uncertainty. Participants rated agreement with 27 statements on a scale from 1 (strongly disagree) to 
5 (strongly agree). The questionnaire has good construct validity, and excellent internal consistency and test-retest reliability (de Bruin et al., 2006; Norton, 2005).

\subsubsection{State measures}

Inspiratory resistances were rated on fear, intensity, and unpleasantness on three Visual Analog Scales (VASs) ranging from 0 (not fearful/intense/unpleasant at all) to 100 (very much fearful/intense/unpleasant). Participants rated how anxious they felt during the previous block (see Section 2.4) on a 0 (not anxious at all) to 100 (very anxious) VAS and threat of resistances within the low category (1), and of resistances within the high category (2) on two 0 (not threatening at all) to 100 (very threatening) VASs.

Pain of the electrocutaneous stimulus was rated on a 0 (no sensation) to 10 (worst pain imaginable) Numeric Rating Scale (NRS). Expectancy of receiving a painful electrocutaneous stimulus if a resistance of the low (1), or of the high category (2) were to be presented next were rated on two 0-100 VASs (certainly not - uncertain - certainly; not anxious at all - very anxious).

\subsection{Procedure}

Upon entering, participants signed informed consent, filled in the trait questionnaires, and maximal inspiratory capacity was tested with the breathing device. Then, during a practice phase, each resistance was presented once and participants were asked to overcome these resistances by increasing breathing effort, and to rate fear, intensity, and unpleasantness of the presented resistance.

Afterwards, the categorization task started. Throughout all phases of this task, inspiratory resistances were presented in quasirandom order, for one breath per resistance. A break was provided in the middle and at the end of each phase described below (dividing each phase into two blocks). 
First, in a category learning phase, a category label was displayed on the screen (4s) and participants memorized whether resistances belonged to the low-intensity $(7,9$, $\left.12,16 \mathrm{cmH}_{2} \mathrm{O}\right)$ or high-intensity $\left(21,28,37,49 \mathrm{cmH}_{2} \mathrm{O}\right)$ category. Resistances were presented six times each and fear, intensity, and unpleasantness were rated on a trial-bytrial basis. The category learning phase existed of two blocks, and each resistance was presented three times per block (48 trials in total).

After the end of this phase, the electrode wristband was attached to the left wrist and the electrocutaneous stimulus was individually calibrated to an intensity that the participant experienced as 'painful and unpleasant, and required some effort to tolerate' (matching a score of 8 on the pain scale) using an ascending method of limits, similar to other studies (e.g., Janssens et al., 2019; Meulders and Vlaeyen, 2019; van Vliet et al., 2018).

Lastly, participants completed a forced-choice categorization task in which they categorized resistances as accurately as possible into the low- or high-intensity category by pressing a button-box (right hand). Which button (left or right) indicated which category (either the low- or high-intensity) was counterbalanced over participants and the respective category labels were displayed on the same side of the screen. After categorizing a resistance, participants rated its fear, intensity, and unpleasantness. This forced-choice task was performed under two conditions (within-subjects), either without threat present (further called the 'safe' condition), or under a threat-of-shock manipulation (further called the 'threat' condition).

In the threat condition, threat-relevance of one of the categories (low- or highintensity; between-subjects) (further called the threat-relevant category) was manipulated by the instruction that "resistances from this category could be followed by the painful stimulus at any time, for a maximum of 10 presentations" (based on the well-validated 
threat-of-shock manipulation of Grillon et al. (2004)). In reality, participants received the painful stimulus six times during the threat condition, immediately following a resistance of the threat-relevant category. Since the presentation of a painful stimulus likely disrupts the processing of the previous resistance, this trial was not analysed. A resistance of the other (threat-irrelevant) category was always presented immediately before or after this trial (random). This trial too was not analysed. Additional resistance pairs were added at fixed time points (after trial 3, 16, and 34 in the first block, and after trial 2, 17, and 33 in the second block). In the safe condition, participants were told that no painful stimuli would be presented and the wristband was removed. In order to keep the number of presented resistances equal between the threat and safe condition, however, one resistance from the low-intensity category, and one resistance from the high-intensity category were added (random order) at the same trials in the safe condition and also were not analysed. Next to these extra resistances, each resistance was presented eight times per condition (four times per block, 152 trials in total). Figure 1 shows the block structure in the threat forced-choice condition (block 1).

Participants rated general anxiety and threat of inspiratory resistances (in each category) in the middle and at the end of the category learning phase and both conditions of the forced-choice categorization phase (threat and safe) (six times in total). Expectancy and fear of the electrocutaneous stimulus were rated at eight time points during the threat condition of the forced-choice phase (after trial 10, 19, 29, and 38 of each block, see Figure 1). Pain of the electrocutaneous stimulus was rated once at the end of calibration, once at the start of the threat condition, and once at the end of this condition. 


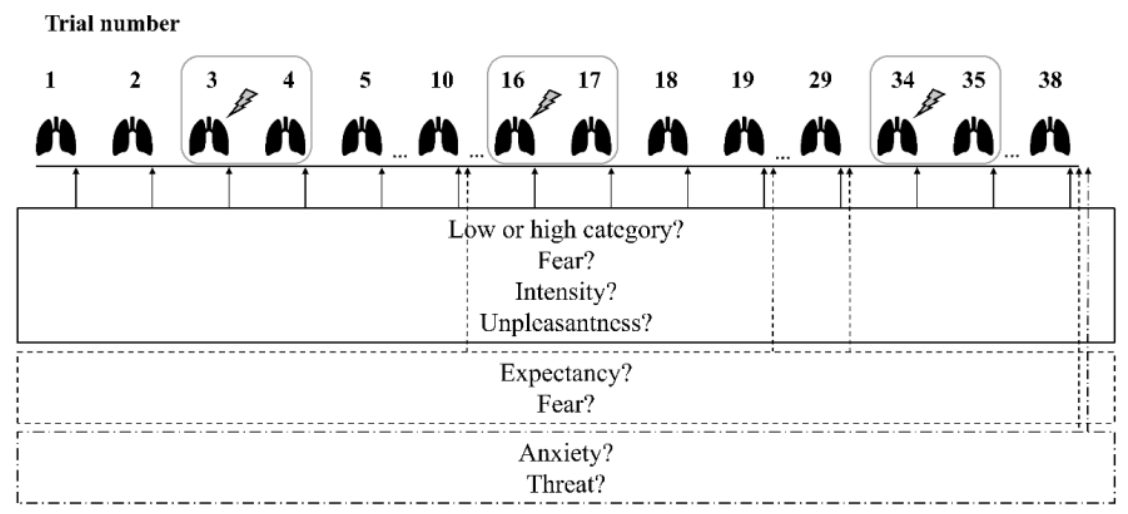

Figure 1. Block structure in the forced choice task (block 1). Lungs: resistance presentation. Grey boxes: extra resistance pairs (random order), one of which was followed by an electrocutaneous stimulus (bolts) in the threat (but not safe) condition.

2.5 Statistical analyses

\subsubsection{Data exclusion}

Individuals who did not differentiate between the threat-relevant and threat-irrelevant category in expectancy to receive a painful stimulus were excluded based on the following precision criterion:

$\frac{\text { mean expectancy threat-relevant category }}{\text { mean expectancy threat-relevant category }+ \text { mean expectancy threat-irrelevant category }} * 100$

We excluded individuals scoring $<70 \%$ on this criterion, that is, those individuals who did not score expectancy of receiving a painful stimulus after a resistance from the threatrelevant category more than twice as high as expectancy after a resistance from the threatirrelevant category. This relative approach was based on information processing theory, that defines precision as the proportion of actual positives (expecting painful a stimulus 
after threat-relevant resistance) that are correctly identified as such (expecting a painful stimulus irrespective of the resistance) (Olson and Delen, 2008, p. 137-138).

\subsubsection{Sample characteristics}

Group differences in age, body mass index (BMI), negative affect, fear of suffocation, intolerance of uncertainty and educational level were examined using independent samples t-tests and a Pearson Chi-square test. It should be noted, however, that we have insufficient power to test for equality and, therefore, results from these tests are thus only approximations.

\subsubsection{Threat manipulation}

Group differences in the intensity of the electrocutaneous stimulus were tested with an independent samples t-test. We also tested whether the painful stimulus was rated as level 8 on the pain scale (painful and unpleasant, and requiring some effort to tolerate) during calibration (one sample t-test) and whether this changed throughout the session (repeated measures (RM) ANOVA), with pain ratings after calibration, before the threat condition, and after the threat condition as levels of the within-subjects variable.

To examine whether the threat manipulation increased general anxiety, we compared mean anxiety in the threat condition to that in the safe condition in a mixeddesign RM-ANOVA, with group as the between-subjects variable. Likewise, to examine whether the threat manipulation significantly increased threat of resistances in the threatrelevant category (low- or high-intensity category, between-subjects), we compared mean threat of resistances in the threat-relevant category in the threat and the safe condition in one ANOVA per between-subjects condition.

It should be noted, however, that we have insufficient power to test for equality and, therefore, results from these tests are thus only approximations. 
2.5.4 Main research questions

All trial-by-trial categorizations and fear, intensity, and unpleasantness ratings made during the forced-choice phases were included in the analyses, except for those made in the extra resistance pairs. SPSS 25 was used to analyse ratings, the stats and lme4 packages in $\mathrm{R}$ were used to analyse binary categorization responses and response times, respectively (Bates et al., 2015). Greenhouse-Geisser and Bonferroni corrections were used when necessary.

2.5.4.1 Research question 1 - Impact of categorical prior beliefs on interoception: perceived sensations within vs. between categories.

To test whether perceived differences between interoceptive stimuli within categories were smaller than between equidistant stimuli at the category border, and whether this effect would be stronger with higher levels of negative affect, fear of suffocation, and intolerance of uncertainty, we calculated the percentage change in mean ratings between adjacent resistances (e.g., $\left[\frac{\text { mean rating resistance } 2 \text { - mean rating resistance } 1}{\text { mean rating resistance } 1}\right] * 100$ ) per rating scale (fear, intensity, and unpleasantness) and condition (threat and safe forced-choice phase) separately. Then, we computed the average percentage change within each category (Corneille et al., 2002; Petersen et al., 2014). Per rating scale, we compared perceived within-category differences to perceived between-category differences in a 3 (percentage change: within the low-intensity category, between categories (i.e., between resistance 45), and within the high-intensity category) x 2 (group: low- or high-intensity category is threat-relevant) x 2 (condition: threat or safe) mixed-design RM-ANCOVA, with negative affect, fear of suffocation, and intolerance of uncertainty as covariates (grand 
mean centred), by testing a planned quadratic contrast across three levels of percentage change.

2.5.4.2 Research question $2 \mathrm{a}$ - Impact of threat-relevant categorical prior belief on interoception: perceived sensations within categories.

To test whether threat-relevance of a categorical prior belief increases its impact on perceived sensations within categories, we ran one RM-ANOVA contrast per rating scale (fear, intensity, and unpleasantness) with average percentage change within the threatrelevant category in the threat versus safe condition as levels of the within-subjects variable and negative affect, fear of suffocation, and intolerance of uncertainty as covariates (grand mean centred). Since the threat-relevant category differed betweensubjects, separate RM-ANOVAs were run for between-subjects conditions (threatrelevant category: low- or high-intensity). We hypothesized that perceived differences would be smaller in the threat than in the safe condition.

2.5.4.3 Research question $2 \mathrm{~b}$ - Impact of threat-relevant categorical prior belief on interoception: between-category discrimination.

To test whether threat related to a categorical prior belief changed the degree of betweencategory discrimination, categorization responses were analysed in a logistic regression analysis. We added resistance, condition (threat or safe), group (threat-relevant category: low- or high-intensity), and all their interactions as predictors. Furthermore, we added negative affect, fear of suffocation, and intolerance of uncertainty and their interaction effects with resistance as covariates. For more details about the analysis, see Supplemental Material. We examined whether the steepness of the regression slope, or between-category discrimination, was different between threat and safe conditions, whether this effect was different depending on which category was threat-relevant, and whether differences in between-category discrimination were related to negative affect, 
fear of suffocation or intolerance of uncertainty. We expected stronger between-category discrimination in the threat (compared to the safe) condition.

2.5.4.4 Research question 2c - Impact of threat-relevant categorical prior belief on interoception: subjective category border.

Here we examined the effects of condition, condition $\mathrm{x}$ group, negative affect, fear of suffocation, and intolerance of uncertainty on the threshold to classify resistances in a certain category (i.e., on the category border). The intercept of a logistic regression indicates the midpoint of its curve, and therefore the estimated resistance intensity that would be classified in the low- and high-intensity category in exactly $50 \%$ of trials. A lower intercept indicates a tendency to identify resistances as being from the highintensity category at a lower resistance intensity, that is, a subjective category border located at a lower resistance intensity. We compared $95 \%$ profile confidence intervals of the intercept under the full model (including the same factors as those described in Section 2.5.4.3) to those under a simple model in which the predictor of interest was removed (non-overlap indicating significance). We expected that threat would increase categorization in the threat-relevant category, or lead to more categorization into the highintensity category (due to more conceptual overlap between the low- and high-intensity category by manipulating threat of the low-intensity category).

\subsection{Secondary research question - Response times}

We explored differences in response times between the threat and safe condition, between correct and error responses in categorization, and the interaction between condition and response. We ran a multilevel gamma regression analysis, in which resistance, condition (threat or safe), response (correct or error) and all their interaction effects were added as 
fixed effects. We allowed a random intercept per participant. We hypothesized that response times would be shorter in the threat compared to the safe condition, longer for error than correct categorizations, and explored whether the latter was different between threat and safe condition. For details on the preparation of response times and more details on the analysis, see the Supplemental Material.

\section{Results}

3.1 Data exclusion

Seven participants $\left(\mathrm{n}_{\text {low-intensity threat-relevant }}=4, \mathrm{n}_{\text {high-intensity threat-relevant }}=3\right)$ did not reach the $70 \%$ precision criterion (i.e., did not score expectancy of receiving a painful stimulus twice as high following stimuli of the threat-relevant compared to the threat-irrelevant category), and were excluded from further analyses. Visual inspection of the expectancy ratings of these individuals suggests that these participants learned to expect the painful stimulus (expectancy ratings different from zero) but did not differentiate between the threat-relevant and threat-irrelevant category (i.e., generalization of threat to the threatirrelevant category) (see Figure 2). 


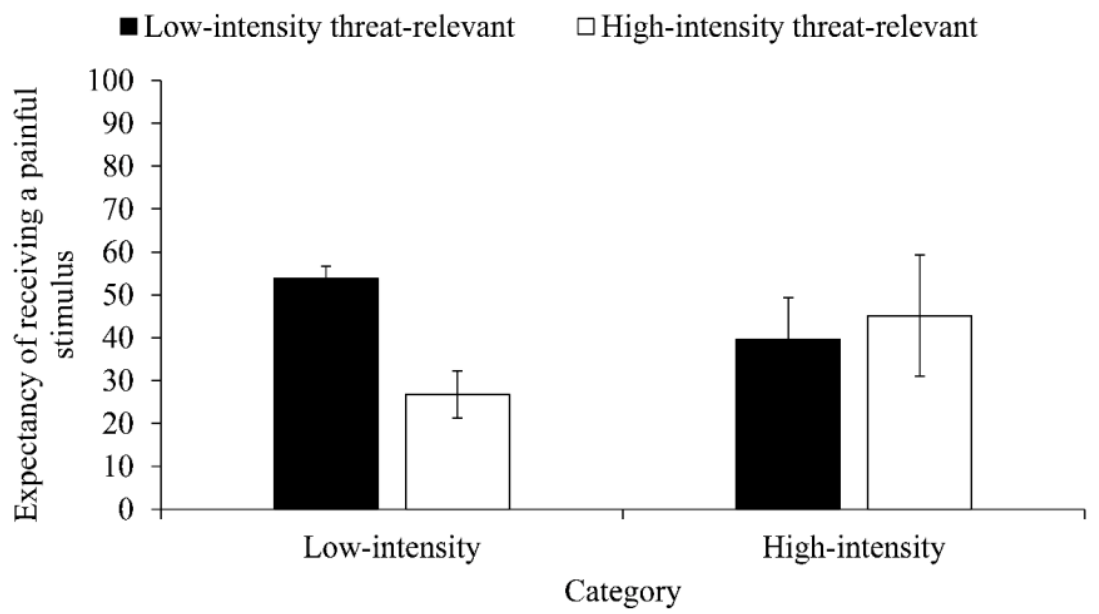

Figure 2. Expectancy of receiving a painful stimulus after a resistance of the low-intensity (left panel) versus the high-intensity category (right panel), for groups that received painful stimuli after resistances of the low-intensity category (black) versus the highintensity category (white). Error bars represent standard errors.

3.2 Sample characteristics

The final sample consisted of 73 participants, divided in two groups differing on the threat-relevant category $\left(n_{\text {low }}=36, n_{\text {high }}=37\right)$. Groups did not differ in age $\left(M_{\text {age }}=20.63\right.$, $\left.S D_{\text {age }}=2.97 ; t(71)=1.95, p=.06, d=.46\right), \mathrm{BMI}\left(M_{\mathrm{BMI}}=22.21, S D_{\mathrm{BMI}}=2.99 ; t(71)=\right.$ $1.46, p=.15, d=.16)$, negative affect $\left(M_{\mathrm{NA}}=23.04, S D_{\mathrm{NA}}=5.48, t(71)=-.66, p=.51\right.$, $d=.15)$, fear of suffocation $\left(M_{\mathrm{FoS}}=6.19, S D_{\mathrm{FoS}}=4.27 ; t(71)=.39, p=.70, d=.09\right)$, or intolerance of uncertainty $\left(M_{\mathrm{IUS}}=63.68, S D_{\mathrm{IUS}}=14.11 ; t(71)=.17, p=.87, d=.04\right)$. Since some cells $(60 \%)$ contained frequencies less than 5, we used a Fisher's exact test instead of Pearson Chi-square. Groups did not differ on educational level $(p=.55, \varphi=$ $.22)$. 
3.3 Threat manipulation

The intensity of the electrocutaneous stimulus did not differ between groups $\left(M_{\text {intensity }}=\right.$ $\left.2.69 \mathrm{~mA}, S D_{\text {intensity }}=1.22 ; t(71)=.16, p=.87, d=.04\right)$. Self-reported pain intensity did not differ from a score of 8 on the pain scale $\left(M_{\text {pain }}=7.96, S D_{\text {pain }}=.31, t(72)=-1.14, p=\right.$ $.26, d=.13)$ and did not change throughout the session $(F(1.25,89.80)=.95, p=.35$, $\eta_{\text {partial }}^{2}=.35$ ), indicating that the stimulus remained 'painful and unpleasant, and requiring effort to tolerate'.

We corrected for heterogeneity of variance in general anxiety ratings with a logarithmic transformation. General anxiety was significantly higher after threat compared to safe blocks $\left(F(1,71)=49.49, p<.001, \eta_{\text {partial }}^{2}=.41\right)$. Threat of the threatrelevant category was significantly higher after threat compared to safe blocks, both in the condition in which the low-intensity category was threat-relevant $(F(1,35)=22.95, p$

$\left.<.001, \eta_{\text {partial }}^{2}=.40\right)$ as in which the high-intensity one was $(F(1,36)=27.55, p<.001$, $\left.\eta_{\text {partial }}^{2}=.43\right)$.

\subsection{Main research questions}

3.4.1 Research question 1 - Impact of categorical prior beliefs on interoception: perceived sensations within vs. between categories.

Significant outliers (data points \pm 3 interquartile range away from the mean of their experimental condition) had virtually no impact on the effects of interest and were therefore included to improve statistical power. Trait negative affectivity, fear of suffocation, and intolerance of uncertainty were not related to the impact of threat on perceived within-category differences (all $p s>.025$ ) and were excluded for further analyses. As depicted in Figure 3, perceived within-category differences were 
significantly smaller than between-category differences in fear (quadratic contrast: $\left.F(1,71)=6.47, p<.025, \eta_{\text {partial }}^{2}=.08\right)$, intensity (quadratic contrast: $F(1,71)=16.95, p<$ $.001, \eta_{\text {partial }}^{2}=.19$ ), as well as unpleasantness ratings (quadratic contrast: $F(1,71)=14.49$, $\left.p<.001, \eta_{\text {partial }}^{2}=.17\right)$. These results thus show that interoceptive sensations are perceived as more similar when they are grouped in the same category compared to perceived differences at the category border.

3.4.2 Research question 2a - Impact of threat-relevant categorical prior belief on interoception: perceived sensations within categories.

Significant outliers (data points \pm 3 interquartile range away from the mean of their experimental condition) were removed from the analyses ( 5 out of 73 data points for fear, 4 for intensity, and 4 for unpleasantness). Trait negative affectivity, fear of suffocation, and intolerance of uncertainty were not related to the impact of threat on perceived withincategory differences (all $p \mathrm{~s}>.025)$ and were excluded for further analyses.

We found that perceived differences in fear within the threat-relevant category were significantly smaller in the threat, compared to the safe condition, but only when the low-intensity category was threat-relevant (low-intensity is threat-relevant: $F(1,32)=$ $7.82, p<.01, \eta_{\text {partial }}^{2}=.20$; high -intensity is threat-relevant: $F(1,34)=1.97, p=.17, \eta_{\text {partial }}^{2}$ $=.06$ ). With regard to intensity ratings, we found smaller perceived differences in the threat-relevant category during the threat (compared to the safe) condition when the highintensity category $\left(F(1,33)=7.413, p<.05, \eta_{\text {partial }}^{2}=.18\right)$, but not when low-intensity category was threat-relevant $\left(F(1,34)=.40, p=.53, \eta_{\text {partial }}^{2}=.01\right)$. We did not find such effects in unpleasantness perception (low-intensity is threat-relevant: $F(1,32)=3.07, p=$ $.09, \eta_{\text {partial }}^{2}=.09$; high-intensity is threat-relevant: $\left.F(1,35)=.80, p=.38, \eta_{\text {partial }}^{2}=.02\right)$. 
These results suggest that perceived within-category detail decreased in the threatrelevant category for fear ratings when the low-intensity category was threat-relevant and for intensity ratings when the high-intensity category was threat-relevant. 
..... Safe —Threat
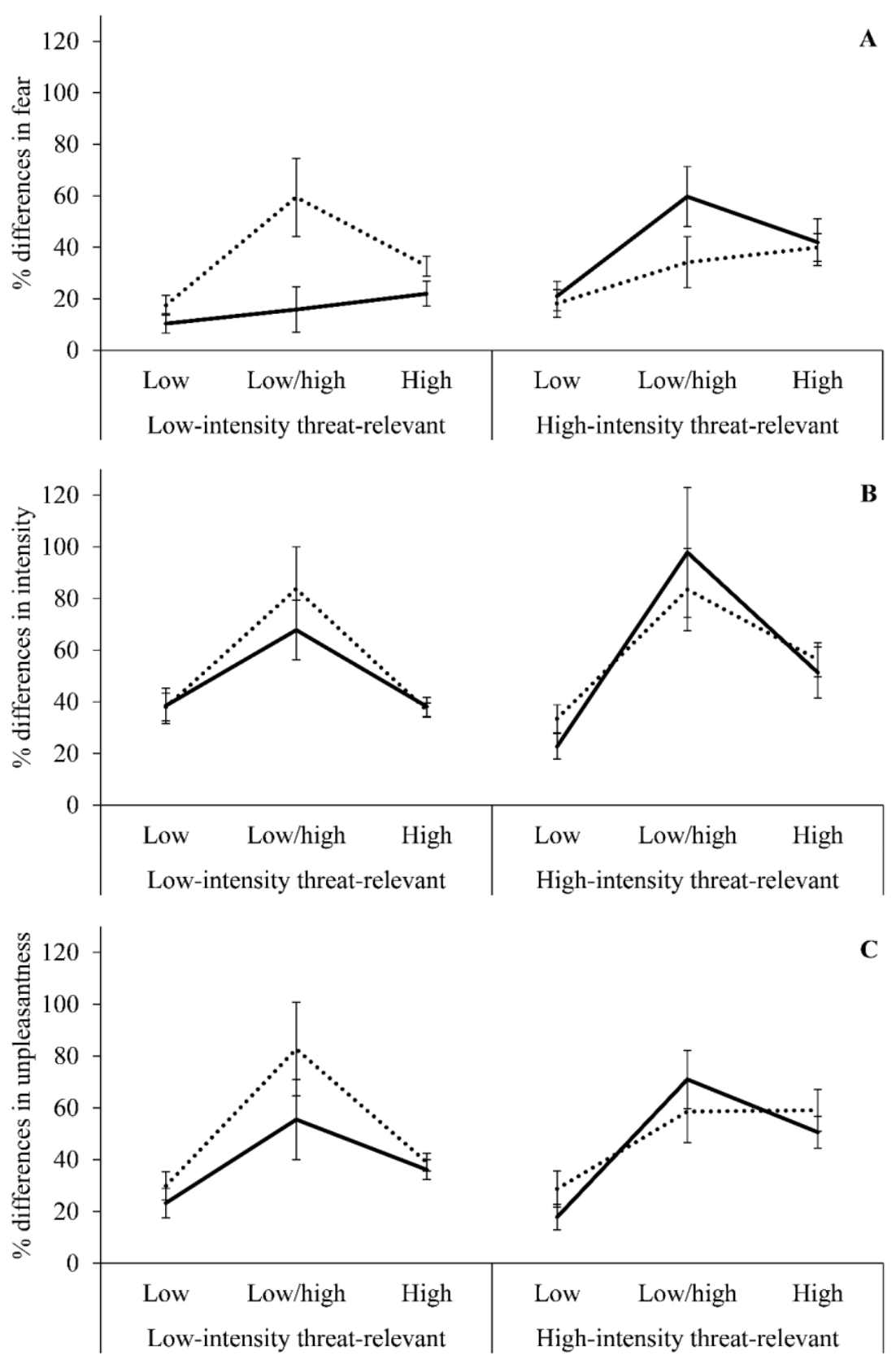
Figure 3. Percentage differences in fear (A), intensity (B), and unpleasantness (C) ratings between resistances within the low-intensity category, at the category border (low/high), and within the high-intensity category, for groups that received painful stimuli after resistances of the low-intensity (left panel) versus high-intensity category (right panel), in the safe (dotted line) and threat (solid line) forced-choice condition. Error bars represent standard errors.

3.4.3 Research question $2 \mathrm{~b}$ - Impact of threat-relevant categorical prior belief on interoception: between-category discrimination.

As shown in Figure 4, between-category discrimination (steepness of regression slopes) did not differ between conditions $\left(\mathrm{OR}_{\text {resistance } \mathrm{x} \text { condition }}=1.01, p=.96,95 \% \mathrm{CI}_{\mathrm{OR}}[.69\right.$, $1.48])$, in neither of the groups $\left(\mathrm{OR}_{\text {resistance } \mathrm{x} \text { condition } \mathrm{x} \text { group }}=.74, p=.30,95 \%\right.$ CIoR $[.42$, 1.31]). Between-category discrimination was stronger in individuals high in fear of suffocation $\left(\mathrm{OR}_{\text {resistance } x \text { FOS }}=1.20, p<.05,95 \% \mathrm{CI}_{\mathrm{OR}}[1.02,1.42]\right)$, but it was not related to negative affectivity $\left(\mathrm{OR}_{\text {resistance } \mathrm{x} \mathrm{NA}}=1.01, p=.96,95 \% \mathrm{CI}_{\mathrm{OR}}[.83,1.22]\right)$, nor intolerance of uncertainty $\left(\mathrm{OR}_{\text {resistance } \mathrm{x} \text { IUS }}=.95, p=.60,95 \% \mathrm{CI}_{\mathrm{OR}}[.80,1.14]\right)$. Thus, the threat manipulation did not impact the strength of between-category discrimination, independent of which category was threat-relevant. Between-category discrimination was, however, stronger in individuals with high fear of suffocation scores.

\subsubsection{Research question 2c - Impact of threat-relevant categorical prior belief} on interoception: subjective category border.

The confidence interval of the intercept under the full model (95\% CI [-0.56,-.27]) was compared to the confidence intervals of the intercept in simpler models (excluding the predictor of interest). Figure 4 shows that the intercept was not impacted significantly by condition (95\% CI [-.46,-.25]), in neither of the groups (95\% CI [-.62,-.47]). Neither fear 
of suffocation, negative affectivity, nor intolerance of uncertainty were associated with the subjective category border $\left(95 \% \mathrm{CI}_{\mathrm{FOS}}[-.56,-.27] ; 95 \% \mathrm{CI}_{\mathrm{NA}}[-.57,-.29] ; 95 \% \mathrm{CI}_{\mathrm{IUS}}\right.$ $[-.56,-.27]$. In summary, the threat manipulation did not have an impact on the subjective category border, independent of whether threat was related to low- or high-intensity sensations. Fear of suffocation, negative affectivity, nor intolerance of uncertainty levels were related to the subjective category border

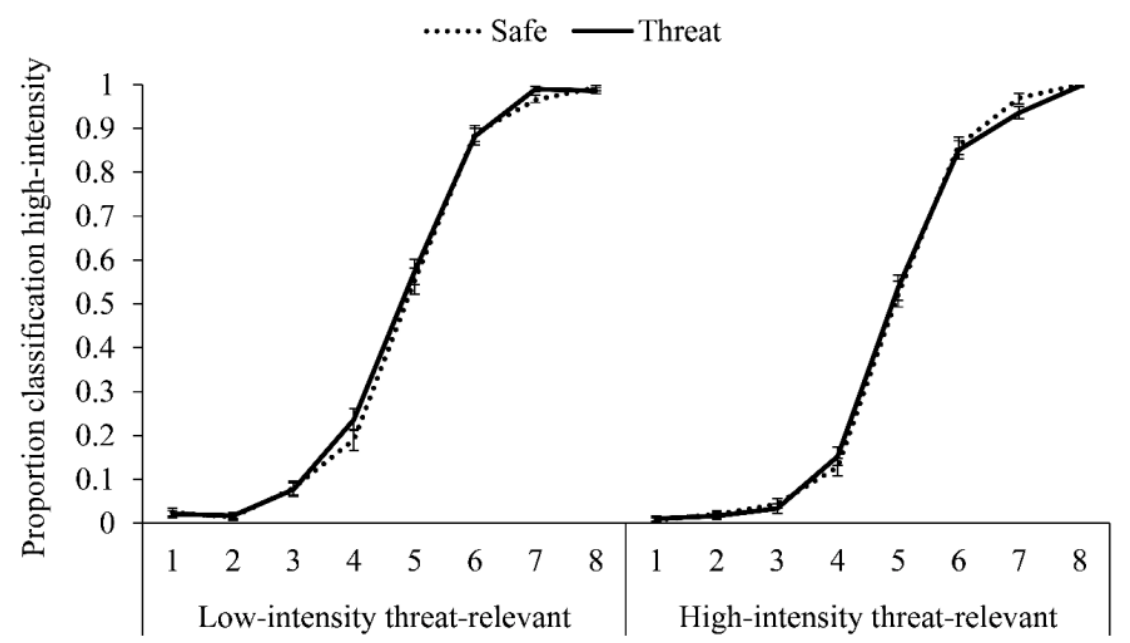

Figure 4. Proportion of trials classified in the high-intensity category in the safe (dotted line) and threat (solid line) forced-choice condition, for groups that received painful stimuli presented after resistances of the low-intensity (left panel) versus the highintensity category (right panel). Error bars represent standard errors.

3.5 Secondary research question: response times

Response times were longer when an error was made, compared to when a resistance was categorized correctly $\left(\beta_{\text {response }}=168.16, p<.0001, d=.58\right)$ and in the threat condition compared to the safe condition $\left(\beta_{\text {condition }}=48.37, p<.0001, d=.15\right)$. These effects did 
not interact $\left(\beta_{\text {response } x \text { condition }}=-7.13, p=.87, d=.03\right)$. Thus, response times were longer when an error was made and in the threat condition.

\section{Discussion}

The aim of this study was to replicate effects of categorization on interoception and to examine whether threat-relevance of categories increases their impact on perceived differences in interoceptive sensations, between-category discrimination, and the subjective category border. We examined interactions with general and specific anxiogenic traits and explored response times.

In accordance with our hypothesis, we found that instigating categorical predictions resulted in smaller perceived differences within, compared to between categories in fear, intensity, and unpleasantness, suggesting that categorical predictions had a profound impact on perceived interoceptive sensations. These findings are consistent with a large body of (exteroceptive) categorization research (e.g., Corneille et al., 2002; Goldstone, 1995; Tajfel and Wilkes, 1963) that found that category membership significantly changes stimulus perception, and extend those found in earlier interoceptive categorization studies, in which category labels were shown on screen immediately before ratings were made (Petersen et al., 2014; van der Meulen et al., 2017), to persistent interoceptive categorization effects even when category labels are no longer shown (i.e., have to be retrieved from memory). Our effects may have been enhanced by increased attention focus on between-category (compared to within-category) differences in order to perform better on the categorization task, but they nevertheless suggest 1) category learning, 2) the significance of the (arbitrary) category border, and 3) the impact of categories on perceived interoceptive sensations. These top-down effects on interoception may be clinically important, since the information frame used to diagnose and discuss 
sensations and symptoms with patients may induce profound changes in the perception thereof (Petersen et al., 2014).

Furthermore, the threat manipulation successfully increased the threat value of resistances in the threat-relevant category, as well as general anxiety in the threat compared to the safe condition. Partially confirming our hypotheses, we found that the threat manipulation resulted in smaller within-category perceived differences in fear, but only when the low-intensity category was threat-relevant. For intensity perception, we found the opposite effect, that is, smaller perceived differences within the threat-relevant category only when the high-intensity category was threat-relevant. These results may point to different effects of threat learning depending on the initial characteristics of categorical prior beliefs (i.e., differences in category attributes). On the one hand, when sensations that differ in intensity are predictive of the occurrence of an aversive outcome, focusing on differences between categories would help detection of, and responding to possible threat. On the other hand, when differences in intensity are of little help in predicting the occurrence of an aversive event (i.e., either an electrocutaneous stimulus or a high-intensity inspiratory resistance), such change in focus may not occur, resulting in a lack of difference between the threat and safe condition in perception of withincategory detail when the low-intensity category is threat-relevant. Similar effects have indeed been found in a respiratory fear conditioning study (Schroijen et al., 2015), and suggest that interoceptive processing flexibly adapts to contextual factors (such as threat) in healthy individuals. The simultaneous loss of detail in fear-relevant information in the low-intensity category likely is a consequence of the threat manipulation, increasing fear of low-intensity stimuli to a similar level (and thus a change in category meaning), whereas differences in fear of high-intensity stimuli may have been smaller due to higher 
baseline fear inherent to high-intensity respiratory resistances (Miller and Davenport, 2015; Pappens et al., 2011, 2010).

Despite affecting within-category perception, threat did not result in more accurate discrimination between threat-relevant and -irrelevant stimulation, nor did it induce a tendency to identify threat-irrelevant stimuli as being threat-relevant. In other words, threat induced changes in perception of respiratory stimuli, but not in choice behaviour. Since the strength of between-category discrimination is impacted by the perceptual similarity between categories, or category overlap (Lynn and Barrett, 2014), our results suggest that threat did not change perceived similarity between categories. Again, taking into account baseline differences in fear related to resistance intensity (Miller and Davenport, 2015; Pappens et al., 2011, 2010), however, threat learning related to low-intensity stimuli likely results in more profound and faster fear learning due to higher surprise - or prediction error (Rescorla and Wagner, 1972). This may have increased perceptual similarity between the low- and high-intensity category when lowintensity stimuli were threat-relevant, resulting in worse between-category discrimination, whereas the opposite may be true when high-intensity stimuli are made threatening, and thus an overall lack of effect. In addition, the lack of differences in the subjective category border probably is due to relatively low behavioural relevance of categorization in our study. Choice behaviour is impacted both by the likelihood of signals and noise (i.e., threat-relevant vs. -irrelevant stimulation), as by the costs associated with missing vs. wrongly identifying threat-relevant stimuli (Lynn and Barrett, 2014). The lack of difference in the subjective category border thus indicates that threat in our study did not change estimated likelihood of threat-(ir)relevant stimuli, nor costs associated with categorization in the threat-(ir)relevant category. Indeed, the aversive stimulus followed threat-relevant resistances in only 6 out of 38 presentations, were short 
(instead of long-lasting or chronic), and had little real-life consequences (i.e., costs), which may be insufficient to change estimated likelihood (i.e., precision) or perceived costs of existing predictions (i.e., generate too little/small prediction errors), especially in healthy, non-anxious individuals (Rescorla and Wagner, 1972).

Individual differences in negative affect and intolerance of uncertainty did not interact with any of the effects. Fear of suffocation scores were, however, positively associated with stronger between-category discrimination. High scores on general anxiogenic traits usually are related to a stronger decoupling of subjectively perceived sensations and objective physiology (Katon et al., 2007; Li et al., 2008), especially in a negative affective context (Bogaerts et al., 2005; Van den Bergh et al., 2004). Since scores on negative affect and intolerance of uncertainty in our sample fell into the normal range (de Bruin et al., 2006; Watson et al., 1988), it is possible that we may only find the expected effects in individuals scoring high on these individual difference variables. Conversely, it has also been proposed that modality- or symptom-specific fears (such as fear of suffocation), rather than general anxiogenic traits, are related to biased perception of respiratory stimulation (Janssens et al., 2011), similar to models of pain (Leeuw et al., 2007) and fatigue perception (Lenaert et al., 2018). Unfortunately, fear of suffocation scores were remarkably low in our sample (Radomsky et al., 2001; Van Diest et al., 2010). Participants knew beforehand that the study involved inspiratory resistances, which likely induced a sampling bias (Karos et al., 2018). Nevertheless, higher fear of suffocation was related to stronger between-category discrimination, suggesting that category learning in individuals scoring high on fear of suffocation increased perceptual dissimilarity (i.e., decreased category overlap) between low- and high-intensity sensations. Low perceptual overlap between interoceptive categories - and thus more rigid categorization - is only problematic, however, when category-based inferences (about causes, consequences, and 
coping behaviour) are inaccurate. In other words, we consider low perceptual overlap as a vulnerability factor for low correspondence between peripheral physiology and subjectively reported sensations. Based on earlier research, we can speculate that inaccurate categories could develop through repeated interoceptive fear conditioning experiences (Zaman et al., 2018, 2017).

As a secondary result, we found that response times were slower for classification errors compared to correct classification, and in the threat compared to the safe condition. Although response times are usually faster in fearful situations (Mohanty and Sussman, 2013; Romero-Rebollar et al., 2016), slower response times on error trials have been found in tasks that are difficult and emphasize accuracy instead of speed (Luce (1986) in Ratcliff and Rouder, 1998; Swensson, 1972). Slower response times on error trials and inthe threat condition could thus be understood from a higher cognitive load as a result of task complexity (i.e., the presentation of an additional stimulus) (Schvaneveldt, 1969) and/or from response inhibition to avoid errors (Aylward and Robinson, 2017; Hu et al., 2012; Mkrtchian et al., 2017). Since task instructions emphasized accuracy (instead of speed), slower response times in the threat condition may indicate task motivation (Leotti and Wager, 2010), especially since participants had no control over the occurrence of the electrocutaneous stimulus (e.g., the option to avoid it). Such interpretation is in accordance with the lack of differences in between-category discrimination and the subjective category border. Possibly, we would find the expected effects (i.e., fast responding, but impaired discrimination between threatening and safe interoceptive stimuli) in anxious individuals, who are known to show impaired inhibitory control in response to fear, and generalization of fear to safety cues (Duits et al., 2015; Dymond et al., 2015; Thayer and Lane, 2000). 
The present study has some limitations. First, although we aimed to investigate an adaptive mechanism (that we believe is present in everyone, but can become maladaptive in some), our results may not generalize to individuals scoring higher on body-related fears, such as patients with medically unexplained symptoms, nor to a male population. Indeed, both interoceptive ability as anxiety are different between men and females (Grabauskaite et al., 2017; McLean and Anderson, 2009; Miller and Davenport, 2015), and between medically unexplained symptom patients and healthy controls (Duschek et al., 2017; Henningsen et al., 2003; Kroenke, 2003; Ricciardi et al., 2016). In addition, we excluded individuals who showed impairments in threat learning. However, this impairment may be a vulnerability factor in the development of medically unexplained symptoms or anxiety disorders related to interoceptive stimulation, such as panic disorder. Second, a stronger prediction error induction may be necessary to induce changes in category precision (by presenting more threat-learning trials, using a stronger threat manipulation, focusing on threat-relevance of low-intensity stimuli only, and/or testing highly anxious individuals) (Duits et al., 2015; Dymond et al., 2015). Third, aversive stimuli were from a different modality than interoceptive stimuli (proprioceptive versus respiratory). It is unclear whether benign sensations that predict an aversive outcome are a good proxy for the averseness that is inherent to some interoceptive sensations (e.g., disease symptoms). Fourth, in our study, we cannot disentangle effects of predictions and attention on interoception. Future research could answer this question by adding a between-subjects condition in which participants memorize dimensional instead of categorical labels. Lastly, participants were instructed to focus on accuracy (vs. speed), which may have reduced effects of the threat manipulation on our outcomes.

4.1 Conclusion 
We found that categorical predictions had a profound impact on perceived interoceptive sensations, thereby replicating earlier research. We did find some evidence for the impact of threat on perceived characteristics of stimuli (with the direction of these effects depending on whether interoceptive stimuli of low or high intensity were threat-relevant), but not for changed precision of categorical predictions. These results imply that the perception of respiratory stimuli is impacted strongly by top-down processes such as categorization (e.g., as disease symptoms), and suggest that interoceptive processing in healthy individuals may flexibly adapt to contextual factors (such as threat), resulting in a lack of differences in behaviour. Rigid responding of repeated and/or severe threat to the internal body (which we hypothesize is associated with individuals high in (interoceptive) anxiety), however, may pose individuals at risk for inaccurate interoception and interoceptive illusions (e.g., medically unexplained symptoms and syndromes).

\section{Acknowledgements}

The authors would like to thank Mathijs Franssen for his technical assistance and help with data processing, and Sarah Hoeylaerts, Annelies Vermeulen, and Anabella Dobre for their help with data collection. This work was supported by the Center for Excellence on Generalization Research [GRIP*TT, KU Leuven grant PF/10/005]; and the Asthenes long-term structural funding by the FWO-Vlaanderen, Flemish Government, Belgium under Grant [METH/15/011].

\section{Disclosure statement}

Declaration of interest: none.

\section{References}

Aylward, J., Robinson, O.J., 2017. Towards an emotional "stress test": A reliable, non- 
subjective cognitive measure of anxious responding. Sci. Rep. 7, 40094.

https://doi.org/10.1038/srep40094

Bar-Haim, Y., Lamy, D., Pergamin, L., Bakermans-Kranenburg, M.J., van IJzendoorn,

M.H., 2007. Threat-related attentional bias in anxious and nonanxious individuals: A meta-analytic study. Psychol. Bull. 133, 1-24. https://doi.org/10.1037/00332909.133.1.1

Barrett, L.F., 2017. The theory of constructed emotion: An active inference account of interoception and categorization. Soc. Cogn. Affect. Neurosci. 12, 1-23. https://doi.org/10.1093/scan/nsw154

Barsky, A.J., Orav, E.J., Bates, D.W., 2005. Somatization increases medical utilization and costs independent of psychiatric and medical comorbidity. Arch. Gen. Psychiatry 62, 903-910. https://doi.org/10.1001/archpsyc.62.8.903

Bates, D., Mächler, M., Bolker, B., Walker, S., 2015. Fitting linear mixed-effects models using lme4. J. Stat. Software, Artic. 67. https://doi.org/10.18637/jss.v067.i01

Bermingham, S.L., Cohen, A., Hague, J., Parsonage, M., 2010. The cost of somatisation among the working-age population in England for the year 2008-2009. Ment. Health Fam. Med. 7, 71-84.

Bogaerts, K., Notebaert, K., Van Diest, I., Devriese, S., De Peuter, S., Van den Bergh, O., 2005. Accuracy of respiratory symptom perception in different affective contexts. J. Psychosom. Res. 58, 537-543. https://doi.org/10.1016/j.jpsychores.2004.12.005

Clarke, D.D., 2016. Diagnosis and treatment of medically unexplained symptoms and chronic functional syndromes. Fam. Syst. Heal. 34, 309-316.

https://doi.org/10.1037/fsh0000228 
Corneille, O., Klein, O., Lambert, S., Judd, C.M., 2002. On the role of familiarity with units of measurement in categorical accentuation: Tajfel and Wilkes (1963) revisited and replicated. Psychol. Sci. 13, 380-383. https://doi.org/10.1111/14679280.00468

de Bruin, G.O., Rassin, E., van der Heiden, C., Muris, P., 2006. Psychometric properties of a Dutch version of the Intolerance of Uncertainty Scale. Neth. J. Psychol. 62, 87-92. https://doi.org/10.1007/BF03061055

Duits, P., Cath, D.C., Lissek, S., Hox, J.J., Hamm, A.O., Engelhard, I.M., Van Den Hout, M.A., Baas, J.M.P., 2015. Updated meta-analysis of classical fear conditioning in the anxiety disorders. Depress. Anxiety 32, 239-253. https://doi.org/10.1002/da.22353

Duschek, S., Montoro, C.I., Reyes Del Paso, G. a., 2017. Diminished interoceptive awareness in fibromyalgia syndrome. Behav. Med. 43, 100-107. https://doi.org/10.1080/08964289.2015.1094442

Dymond, S., Dunsmoor, J.E., Vervliet, B., Roche, B., Hermans, D., 2015. Fear generalization in humans: Systematic review and implications for anxiety disorder research. Behav. Ther. 46, 561-582. https://doi.org/10.1016/j.beth.2014.10.001

Engelen, U., De Peuter, S., Victoir, A., Van Diest, I., Van den Bergh, O., 2006. Verdere validering van de Positive and Negative Affect Schedule (PANAS) en vergelijking van twee Nederlandstalige versies. Gedrag en Gezondh. 34, 89-102. https://doi.org/https://doi.org/10.1007/BF03087979

Geisser, M.E., Casey, K.L., Bruksch, C.B., Ribbens, C.M., Appleton, B.B., Crofford, L.J., 2003. Perception of noxious and innocuous heat stimulation among healthy women and women with fibromyalgia: association with mood, somatic focus, and catastrophizing. Pain 102, 243-250. https://doi.org/10.1016/S0304- 
3959(02)00417-7

Goldstone, R.L., 1995. Effects of categorization on color perception. Psychol. Sci. 6, 298-304. https://doi.org/10.1111/j.1467-9280.1995.tb00514.x

Grabauskaite, A., Baranauskas, M., Griskova-Bulanova, I., 2017. Interoception and gender: What aspects should we pay attention to? Conscious. Cogn. 48, 129-137. https://doi.org/10.1016/j.concog.2016.11.002

Grillon, C., Baas, J.P., Lissek, S., Smith, K., Milstein, J., 2004. Anxious responses to predictable and unpredictable aversive events. Behav. Neurosci. 118, 916-924. https://doi.org/10.1037/0735-7044.118.5.916

Haller, H., Cramer, H., Lauche, R., Dobos, G., 2015. Somatoform disorders and medically unexplained symptoms in primary care - A systematic review and metaanalysis of prevalence. Dtsch. Arztebl. Int. 112, 279-287.

https://doi.org/10.3238/arztebl.2015.0279

Henningsen, P., Gündel, H., Kop, W.J., Löwe, B., Martin, A., Rief, W., Rosmalen, J.G.M., Schröder, A., van der Feltz-Cornelis, C., Van den Bergh, O., 2018. Persistent physical symptoms as perceptual dysregulation: A neuropsychobehavioral model and its clinical implications. Psychosom. Med. 80, 422-431. https://doi.org/10.1097/PSY.0000000000000588

Henningsen, P., Zimmermann, T., Sattel, H., 2003. Medically unexplained physical symptoms, anxiety, and depression: A meta-analytic review. Psychosom. Med. 65, 528-533. https://doi.org/10.1097/01.PSY.0000075977.90337.E7

Hiller, W., Fichter, M.M., Rief, W., 2003. A controlled treatment study of somatoform disorders including analysis of healthcare utilization and cost-effectiveness. J. Psychosom. Res. 54, 369-380. https://doi.org/10.1016/S0022-3999(02)00397-5

Hu, K., Bauer, A., Padmala, S., Pessoa, L., 2012. Threat of bodily harm has opposing 
effects on cognition. Emotion 12, 28-32. https://doi.org/10.1037/a0024345.Threat Janssens, T., De Peuter, S., Stans, L., Verleden, G., Troosters, T., Decramer, M., Van Den Bergh, O., 2011. Dyspnea perception in COPD: Association between anxiety, dyspnea-related fear, and dyspnea in a pulmonary rehabilitation program. Chest 140, 618-625. https://doi.org/10.1378/chest.10-3257

Janssens, T., Meulders, A., Cuyvers, B., Colloca, L., Vlaeyen, J.W.S., 2019. Placebo and nocebo effects and operant pain-related avoidance learning. PAIN Reports 4, e748. https://doi.org/10.1097/pr9.0000000000000748

Jerndal, P., Ringström, G., Agerforz, P., Karpefors, M., Akkermans, L.M., Bayati, A., Simrén, M., 2010. Gastrointestinal-specific anxiety: An important factor for severity of GI symptoms and quality of life in IBS. Neurogastroenterol. Motil. 22, 646-e179. https://doi.org/10.1111/j.1365-2982.2010.01493.x

Karos, K., Alleva, J.M., Peters, M.L., 2018. Pain, please: An investigation of sampling bias in pain research. J. Pain 19, 787-796. https://doi.org/10.1016/j.jpain.2018.02.011

Katon, W., Lin, E.H.B., Kroenke, K., 2007. The association of depression and anxiety with medical symptom burden in patients with chronic medical illness. Gen. Hosp. Psychiatry 29, 147-155. https://doi.org/10.1016/j.genhosppsych.2006.11.005

Kroenke, K., 2003. Patients presenting with somatic complaints: Epidemiology, psychiatric co-morbidity and management. Int. J. Methods Psychiatr. Res. 12, 3443. https://doi.org/10.1002/mpr.140

Leeuw, M., Goossens, M.E.J.B., Linton, S.J., Crombez, G., Boersma, K., Vlaeyen, J.W.S., 2007. The fear-avoidance model of musculoskeletal pain: Current state of scientific evidence. J. Behav. Med. 30, 77-94. https://doi.org/10.1007/s10865-0069085-0 
Lenaert, B., Boddez, Y., Vlaeyen, J.W.S., van Heugten, C.M., 2018. Learning to feel tired: A learning trajectory towards chronic fatigue. Behav. Res. Ther. 100, 54-66. https://doi.org/10.1016/j.brat.2017.11.004

Leotti, L.A., Wager, T.D., 2010. Motivational influences on response inhibition measures. J. Exp. Psychol. Hum. Percept. Perform. 36, 430-447. https://doi.org/10.1037/a0016802

Li, W., Van Diest, I., De Peuter, S., Bogaerts, K., Oyen, N., Hombroux, N., Van de Woestijne, K., Gallego, J., Van den Bergh, O., 2008. Repeated experiences of air hunger and ventilatory behavior in response to hypercapnia in the standardized rebreathing test: Effects of anxiety. Biol. Psychol. 77, 223-232. https://doi.org/10.1016/j.biopsycho.2007.10.013

Lissek, S., Powers, A.S., McClure, E.B., Phelps, E.A., Woldehawariat, G., Grillon, C., Pine, D.S., 2005. Classical fear conditioning in the anxiety disorders: A metaanalysis. Behav. Res. Ther. 43, 1391-1424. https://doi.org/10.1016/j.brat.2004.10.007

Lissek, S., Rabin, S.J., McDowell, D.J., Dvir, S., Bradford, D.E., Geraci, M., Pine, D.S., Grillon, C., 2009. Impaired discriminative fear-conditioning resulting from elevated fear responding to learned safety cues among individuals with panic disorder. Behav. Res. Ther. 47, 111-118. https://doi.org/10.1016/j.brat.2008.10.017

Lynn, S.K., Barrett, L.F., 2014. "Utilizing” signal detection theory. Psychol. Sci. 25, 1663-1673. https://doi.org/10.1177/0956797614541991

McLean, C.P., Anderson, E.R., 2009. Brave men and timid women? A review of the gender differences in fear and anxiety. Clin. Psychol. Rev. 29, 496-505.

https://doi.org/10.1016/j.cpr.2009.05.003 
Merz, C.J., Kinner, V.L., Wolf, O.T., 2018. Let's talk about sex ... differences in human fear conditioning. Curr. Opin. Behav. Sci. 23, 7-12.

https://doi.org/10.1016/j.cobeha.2018.01.021

Meulders, A., Vlaeyen, J.W., 2019. The effect of differential spatiotopic information on the acquisition and generalization of fear of movement-related pain. PeerJ 7, e6913. https://doi.org/10.7717/peerj.6913

Miller, S., Davenport, P.W., 2015. Subjective ratings of prolonged inspiratory resistive loaded breathing in males and females. Psychophysiology 52, 90-97. https://doi.org/10.1111/psyp.12297

Mkrtchian, A., Roiser, J.P., Robinson, O.J., 2017. Threat of shock and aversive inhibition: Induced anxiety modulates Pavlovian-instrumental interactions. J. Exp. Psychol. Gen. 146, 1694-1704. https://doi.org/10.1037/xge0000363

Mohanty, A., Sussman, T.J., 2013. Top-down modulation of attention by emotion. Front. Hum. Neurosci. 7, 102. https://doi.org/10.3389/fnhum.2013.00102

Nettleton, S., 2006. "I just want permission to be ill": Towards a sociology of medically unexplained symptoms. Soc. Sci. Med. 62, 1167-1178.

https://doi.org/10.1016/j.socscimed.2005.07.030

Nijs, J., Roussel, N., Van Oosterwijck, J., De Kooning, M., Ickmans, K., Struyf, F., Meeus, M., Lundberg, M., 2013. Fear of movement and avoidance behaviour toward physical activity in chronic-fatigue syndrome and fibromyalgia: State of the art and implications for clinical practice. Clin. Rheumatol. 32, 1121-1129. https://doi.org/10.1007/s10067-013-2277-4

Nimnuan, C., Hotopf, M., Wessely, S., 2001. Medically unexplained symptoms: An epidemiological study in seven specialities. J. Psychosom. Res. 51, 361-367. https://doi.org/10.1016/S0022-3999(01)00223-9 
Norton, P.J., 2005. A psychometric analysis of the Intolerance of Uncertainty Scale among four racial groups. J. Anxiety Disord. 19, 699-707.

https://doi.org/10.1016/j.janxdis.2004.08.002

Olson, D.L., Delen, D., 2008. Advanced data mining techniques, 1st ed, Advanced Data Mining Techniques. Springer-Verlag, Berlin. https://doi.org/10.1007/978-3-540$76917-0$

Pappens, M., Van den Bergh, O., De Peuter, S., Bresseleers, J., Vansteenwegen, D., Van Diest, I., 2010. Defense reactions to interoceptive threats: A comparison between loaded breathing and aversive picture viewing. Biol. Psychol. 84, 98-103. https://doi.org/10.1016/j.biopsycho.2010.02.006

Pappens, M., Van den Bergh, O., Vansteenwegen, D., Van Diest, I., 2011.

Psychophysiological responses to inspiratory resistive loads. Int. J. Psychophysiol.

80, 161-165. https://doi.org/10.1016/j.ijpsycho.2011.02.015

Paulus, M.P., Stein, M.B., 2010. Interoception in anxiety and depression. Brain Struct.

Funct. 214, 451-463. https://doi.org/10.1007/s00429-010-0258-9

Peters, M.L., Vlaeyen, J.W.S., van Drunen, C., 2000. Do fibromyalgia patients display hypervigilance for innocuous somatosensory stimuli? Application of a body scanning reaction time paradigm. Pain 86, 283-292.

https://doi.org/10.1016/S0304-3959(00)00259-1

Petersen, S., Schroijen, M., Mölders, C., Zenker, S., Van den Bergh, O., 2014.

Categorical interoception: Perceptual organization of sensations from inside.

Psychol. Sci. 25, 1059-1066. https://doi.org/10.1177/0956797613519110

Petersen, S., Van Staeyen, K., Vögele, C., Von Leupoldt, A., Van den Bergh, O., 2015a. Interoception and symptom reporting: Disentangling accuracy and bias. Front.

Psychol. 6, 732. https://doi.org/10.3389/fpsyg.2015.00732 
Petersen, S., von Leupoldt, A., Van den Bergh, O., 2015b. Interoception and the uneasiness of the mind: Affect as perceptual style. Front. Psychol. 6, 1408. https://doi.org/10.3389/fpsyg.2015.01408

Radomsky, A.S., Rachman, S., Thordarson, D.S., McIsaac, H.K., Teachman, B.A., 2001. The Claustrophobia Questionnaire. J. Anxiety Disord. 15, 287-297. https://doi.org/10.1016/S0887-6185(01)00064-0

Ratcliff, R., Rouder, J.N., 1998. Modeling response times for two-choice decisions. Psychol. Sci. 9, 347-356. https://doi.org/10.1111/1467-9280.00067

Reid, S., Wessely, S., Crayford, T., Hotopf, M., 2001. Medically unexplained symptoms in frequent attenders of secondary health care: Retrospective cohort study. BMJ 322, 767. https://doi.org/10.1136/bmj.322.7289.767

Rescorla, R.A., Wagner, A.R., 1972. A theory of Pavlovian conditioning: Variations in the effectiveness of reinforcement and nonreinforcement, in: Black, A.H., Prokasy, W.F. (Eds.), Classical Conditioning II: Current Research and Theory. AppletonCentury-Crofts, New York, pp. 64-99.

Ricciardi, L., Ferrazzano, G., Demartini, B., Morgante, F., Erro, R., Ganos, C., Bhatia, K.P., Berardelli, A., Edwards, M., 2016. Know thyself: Exploring interoceptive sensitivity in Parkinson's disease. J. Neurol. Sci. 364, 110-115. https://doi.org/10.1016/j.jns.2016.03.019

Rigoli, F., Pezzulo, G., Dolan, R., Friston, K., 2017. A goal-directed Bayesian framework for categorization. Front. Psychol. 8, 408. https://doi.org/10.3389/fpsyg.2017.00408

Romero-Rebollar, C., Jiménez-Ángeles, L., Dragustinovis-Ruiz, E.A., MedinaBañuelos, V., 2016. Neural modulation in aversive emotion processing: An independent component analysis study. Comput. Math. Methods Med. 2016, 1-10. 
https://doi.org/10.1155/2016/2816567

Schroijen, M., Pappens, M., Schruers, K., Van den Bergh, O., Vervliet, B., Van Diest,

I., 2015. Generalization of fear to respiratory sensations. Behav. Ther. 46, 611-

626. https://doi.org/10.1016/j.beth.2015.05.004

Schvaneveldt, R.W., 1969. Effects of complexity in simultaneous reaction time tasks. J.

Exp. Psychol. 81, 289-296. https://doi.org/10.1037/h0027752

Smith, B.M.E., Haney, E., McDonagh, M., Pappas, M., Daeges, M., Wasson, N., Fu, R., Nelson, H.D., 2015. Treatment of myalgic encephalomyelitis/chronic fatigue syndrome: A systematic review for a National Institutes of health pathways to prevention workshop. Ann. Intern. Med. 162, 841-850.

https://doi.org/10.7326/M15-0114

Swensson, R.G., 1972. The elusive tradeoff: Speed vs accuracy in visual discrimination tasks. Percept. Psychophys. 12, 16-32. https://doi.org/10.3758/BF03212837

Tajfel, H., Wilkes, A.L., 1963. Classification and quantitative judgement. Br. J. Psychol. 54, 101-114. https://doi.org/10.1111/j.2044-8295.1963.tb00865.x

Thayer, J.F., Lane, R.D., 2000. A model of neurovisceral integration in emotion regulation and dysregulation. J. Affect. Disord. 61, 201-216. https://doi.org/10.1016/S0165-0327(00)00338-4

Van den Bergh, O., Winters, W., Devriese, S., Van Diest, I., Vos, G., De Peuter, S., 2004. Accuracy of respiratory symptom perception in persons with high and low negative affectivity. Psychol. Health 19, 213-222. https://doi.org/10.1080/08870440410001675627

Van den Bergh, O., Witthöft, M., Petersen, S., Brown, R.J., 2017. Symptoms and the body: Taking the inferential leap. Neurosci. Biobehav. Rev. 74, 185-203. https://doi.org/10.1016/j.neubiorev.2017.01.015 
van der Meulen, M.A., Anton, F., Petersen, S., 2017. Painful decisions: How classifying sensations can change the experience of pain. Eur. J. Pain 21, 1602-1610. https://doi.org/10.1002/ejp.1061

Van Diest, I., Smits, D., Decremer, D., Maes, L., Claes, L., 2010. The Dutch Claustrophobia Questionnaire: Psychometric properties and predictive validity. J. Anxiety Disord. 24, 715-722. https://doi.org/10.1016/j.janxdis.2010.05.003 van Vliet, C.M., Meulders, A., Vancleef, L.M.G., Vlaeyen, J.W.S., 2018. The Opportunity to Avoid Pain May Paradoxically Increase Fear. J. Pain 19, 12221230. https://doi.org/10.1016/j.jpain.2018.05.003

Watson, D., Clark, L.A., Tellegen, A., 1988. Development and validation of brief measures of positive and negative affect: The PANAS scales. J. Pers. Soc. Psychol. 54, 1063-1070. https://doi.org/https://doi.org/10.1037/0022-3514.54.6.1063

Zaman, J., Madden, V.J., Iven, J., Wiech, K., Weltens, N., Ly, H.G., Vlaeyen, J.W.S., Van Oudenhove, L., Van Diest, I., 2017. Biased intensity judgements of visceral sensations after learning to fear visceral stimuli: A drift diffusion approach. J. Pain 18, 1197-1208. https://doi.org/10.1016/j.jpain.2017.04.011

Zaman, J., Weltens, N., Ly, H.G., Struyf, D., Vlaeyen, J.W.S., Van den Bergh, O.,

Wiech, K., Van Oudenhove, L., Van Diest, I., 2016. Influence of interoceptive fear learning on visceral perception. Psychosom. Med. 78, 248-258.

https://doi.org/10.1097/PSY.0000000000000257

Zaman, J., Wiech, K., Claes, N., Van Oudenhove, L., Van Diest, I., Vlaeyen, J.W.S., 2018. The influence of pain-related expectations on intensity perception of nonpainful somatosensory stimuli. Psychosom. Med. 80, 836-844. https://doi.org/10.1097/psy.0000000000000586

Zamariola, G., Maurage, P., Luminet, O., Corneille, O., 2018. Interoceptive accuracy 
scores from the heartbeat counting task are problematic: Evidence from simple bivariate correlations. Biol. Psychol. 137, 12-17.

https://doi.org/10.1016/j.biopsycho.2018.06.006 